\title{
Review on the Security Threats of Internet of Things
}

\author{
Prajoy Podder \\ Institute of ICT \\ Bangladesh University of \\ Engineering and Technology \\ Dhaka-1205, Bangladesh
}

\author{
M. Rubaiyat Hossain Mondal \\ Institute of ICT \\ Bangladesh University of \\ Engineering and Technology \\ Dhaka-1205, Bangladesh
}

\author{
Pinto Kumar Paul \\ Department of CSE \\ Ranada Prasad Shaha University \\ Narayanganj, Bangladesh
}

\author{
Subrato Bharati \\ Institute of ICT \\ Bangladesh University of \\ Engineering and Technology \\ Dhaka-1205, Bangladesh
}

\begin{abstract}
Internet of Things (IoT) is being considered as the growth engine for industrial revolution 4.0. The combination of IoT, cloud computing and healthcare can contribute in ensuring well-being of people. One important challenge of IoT network is maintaining privacy and to overcome security threats. This paper provides a systematic review of the security aspects of IoT. Firstly, the application of IoT in industrial and medical service scenarios are described, and the security threats are discussed for the different layers of IoT healthcare architecture. Secondly, different types of existing malware including spyware, viruses, worms, keyloggers, and trojan horses are described in the context of IoT. Thirdly, some of the recent malware attacks such as Mirai, echobot and reaper are discussed. Next, a comparative discussion is presented on the effectiveness of different machine learning algorithms in mitigating the security threats. It is found that the k-nearest neighbor $(\mathrm{kNN})$ machine learning algorithm exhibits excellent accuracy in detecting malware. This paper also reviews different tools for ransomware detection, classification and analysis. Finally, a discussion is presented on the existing security issues, open challenges and possible future scopes in ensuring IoT security.
\end{abstract}

\section{Keywords}

Accuracy, IoT, IoMT, Intrusion Detection, Malware, Machine Learning, Ransomware, Threats.

\section{INTRODUCTION}

Many intelligent systems like gadgets and applications are developing day by day based on advanced technology like the Internet of things (IoT). The usage of IoT is increasing day by day because of its importance. IoT has been recently integrated into many gadgets and applications, to make the system linear to rational. This adoption is increasing day by day because of its importance. IoT technology is also effectively influencing our medical science. The healthcare monitoring system is being developed to ensure emergency services to the patients effectively [1]. Some health application is already developed based on IoT such as emergency notification, continuous glucose monitoring (CGM), and computer-assisted rehabilitation. Those software applications are built to solve different aspects of medical issues. Smartphones are the most crucial part of our daily life and the intelligent application uses the sensor of smartphones. They continuously perceive data from the devices using its sensors and try to monitor the subject's health condition [2].
The whole system needs different types of data from the wards and diagnostics equipment. This is to analyze using data mining and to conclude an efficient result for monitoring and tracking purposes $[3,4]$. After that, the intelligent system gains the ability to control health care automatically [5-6].

However, there are some challenges in the integration of IoT technology. Data storage problem, data management problem, exchange of information between devices, security and privacy - these are the main problems that need to be solved first. Cloud computing can be addressed as one of the most effective solutions for all of these problems. A conventional healthcare system is presented in Figure 1 that integrates both IoT and cloud computing in order to provide the facility to access shared medical data and common infrastructure transparently and efficiently.

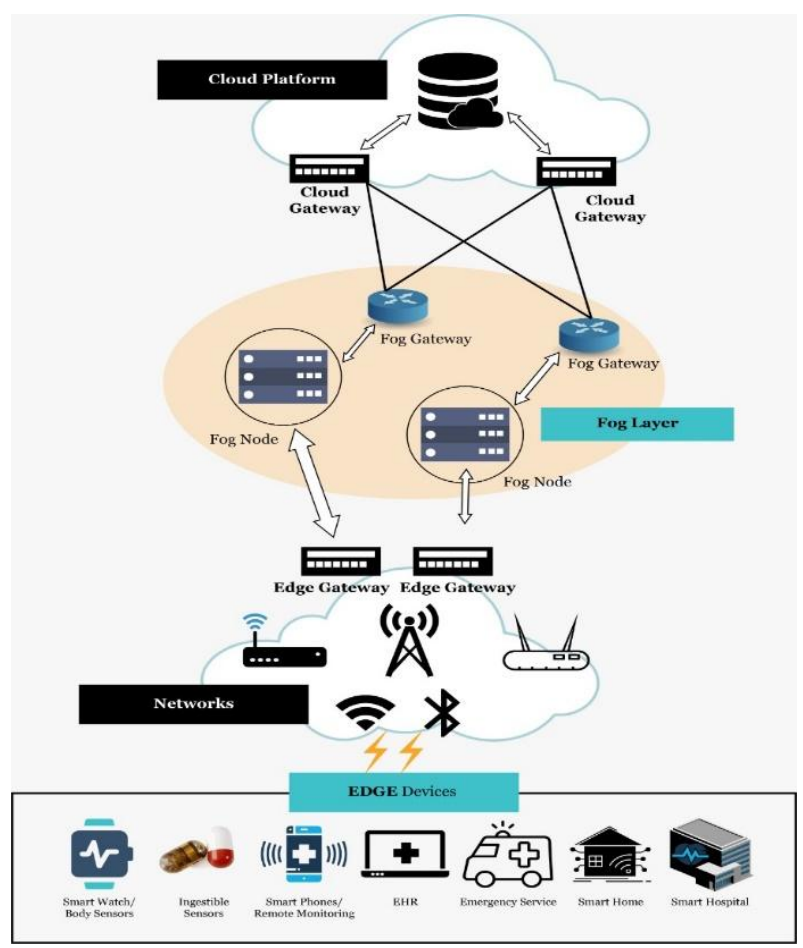

Fig 1: An overview of cloud integrated IoT system

Cloud computing offers computing services i.e. software, databases, servers, data analytics, networking over the Internet to deliver faster expansion, economies of scale and flexible 
resources. In edge devices, fog computing exhibits data analytics, so that it performs real-time processing, reduces costs, and improves the privacy of data. The rise of cloud computing, artificial intelligence and portable devices ensure a solid foundation for the evolution of IoT based healthcare sector.

Medical devices or instruments are also engaged with several wireless communication technologies (i.e., Wi-Fi, bluetooth, etc.) that permit the machine-to-machine communication. It is an environment for Internet of medical things (IoMT) communication. In IoMT, the devices for smart healthcare send data to cloud servers. Several cloud platforms, i.e., Amazon Web Services, may be conducted to store the patients' health related data and to explore the data for health prescriptions and accurate decision making [8].

For rapid deploying and developing the IoT systems, the issues of security in the IoT devices are facing day by day. This increases the probability to launch different types of attacks in the IoMT environment via the Internet. It occurs very crucial issue in the IoMT that controls the smart medical devices with its communication. For example, if an attacker practically obtains the remote control over an IoT based smart medical device, s/he can manipulate the patients' data.

The key motivation behind this work is illustrated as follows. In recent days, IoT devices i.e. smart city, smart home and smart healthcare devices have become the crucial part of our daily life. Since we know it, the users of IoT devices are able to access the data remotely using the Internet [9, 10]. Different entities, such as IoT devices, servers and users, communicate through the Internet. Wi-Fi [11,12], WiMax [13, 14], LTE [15, 16] are the popular forms of using Internet effectively. Light fidelity (LiFi) [11-16] is also an emerging technology for the Internet. OFDM, MIMO systems are playing a vital role in order to establish upgraded wireless communication systems [17-18]. However, there are many forms of security issues in IoT/IoMT communication environment.

The massive scale of IoT based networks carry some new challenges. Existing research papers covered different aspects of IoT such as architecture, communication system, IoT related various applications [19-28], security and privacy [2829]. However, the mainspring of the commercialization of IoT /IoMT and industrial IoT (IIoT) technology is the security and privacy assurance as well as user satisfaction.

Different sorts of IoT malware are continuously emerging. These can easily affect the communication of IoMT. Malware is also used to control the smart medical devices. Different kinds of attacks, i.e., denial of service, replay, password guessing, impersonation and man-in-the-middle (MITM) attacks can get chance to enter in this environment. Usually, the hackers may apply malware to target the Internet based health care devices for entering illegal access or controlling these devices remotely.

To deploy malware in the environment of IoMT, the hackers adopt network of attacker processes such as botnet. Some examples of botnet are Echobot, Mirai, Emotet, Reaper, Necurs and Gamut. These kinds of botnet attacks are also probable in the environment of IoMT and can be permitted to control or hijack an IoMT based smart healthcare device remotely. This can occur several life threatening conditions for the patients. Consequently, people developing in the IoT security domain emerge with novel ideas to protect the environment of IoMT communication against these attacks.
For that reasons, in our work we focus on various types of active IoMT malware and malware programs. The major outcomes of this paper are as follows.

(i) The relation between IoT and cloud computing environment is discussed, and different security requirements of IoT communication environment are illustrated.

(iii) Recent malware attacks such as Mirai, Reaper, Echobot, Emotet, Gamut and Necurs are studied in the context of IoT and IoMT environment.

(iv) The performance of various machine learning techniques for classification and Android malware detection are summarized.

(iv) The effect of ransomware in IoT/IoMT environment is discussed and the existing software tools for ransomware detection are summarized. The remaining part of the manuscript is prepared as follows: Section 2 considers the application of IoT communication environment, while Section 3 discusses security threats. Section 4 introduces various kinds of malware, while Section 5 discusses some of the recent malware. The effectiveness of different machine learning algorithms in recognizing security threats are discussed in Section 6. Ransomware is studied in Section 7, and future research directions are reported in Section 8. The concluding remarks are presented in Section 9.

\section{APPLICATIONS OF IOT COMMNICATION ENVIRONMENT}

In IIoT, sensors and machines in factories, industries are interconnected with each other. Then they provide real-time data over the Internet to the engineers or the manufacturers to increase the industrial processes.

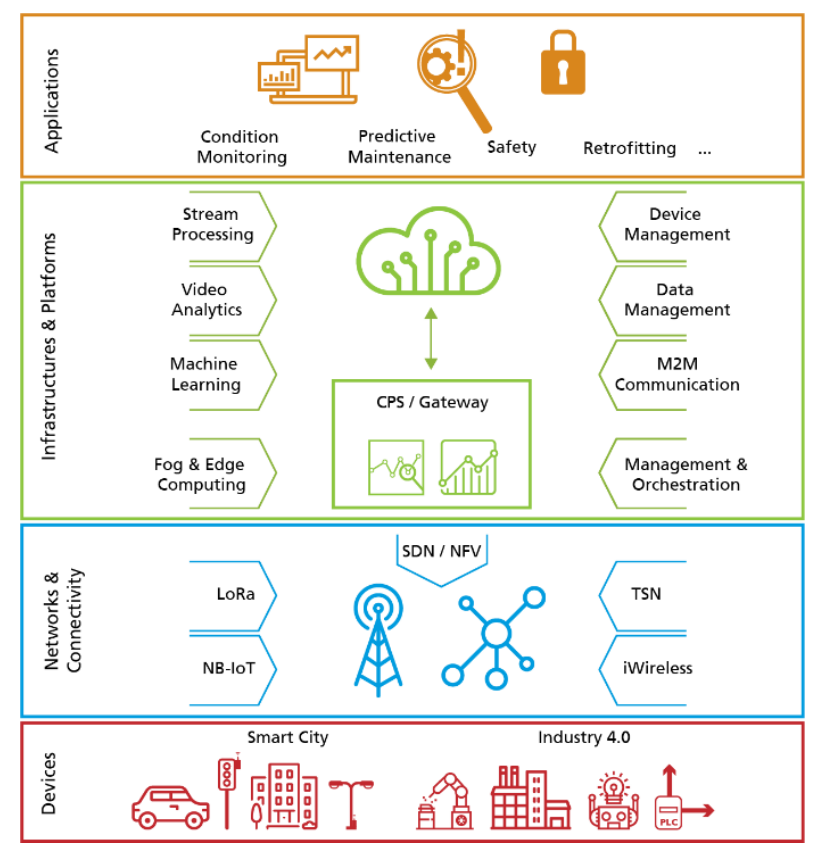

Fig 2: A general overview of IIoT [61]

In the medical or healthcare based systems, reactive healthcare based schemes can be changed into proactive wellness-based schemes using IoMT. These types of systems are particular IoMT based smart healthcare or medical devices monitor as well as send medical data to a cloud server. If a patient's relative or a doctor is attracted in the real-time access of this devices, it can be also accomplished by using the environment of IoT. In this way, IoMT facilitates the analysis, 
processing and access of the suitable medical data.

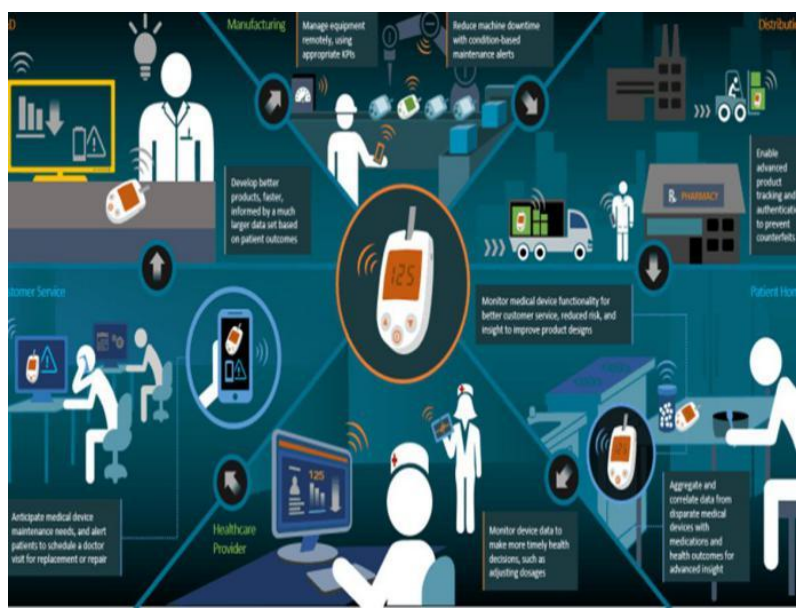

Fig 3: An overview of IoMT [60]

\section{SECURITY AND PRIVACY THREATS IN IOT}

Security and privacy threats are summarized in Figure 4 and Figure 5.

The IoT healthcare applications architecture normally consists of the following layers [22]:
a) Application
b) Communication
c) Device
d) Network
e) Transport.

The percentage rate of affecting the layers are as follows:
a) Application layer (9\%)
b) Communication layer (18\%)
c) Device layer $(42 \%)$
d) Network layer (27\%)
e) Transport layer (4\%).

From the above discussion, it can be said that device layer suffers the highest impact, while the transport layer is affected the lowest.

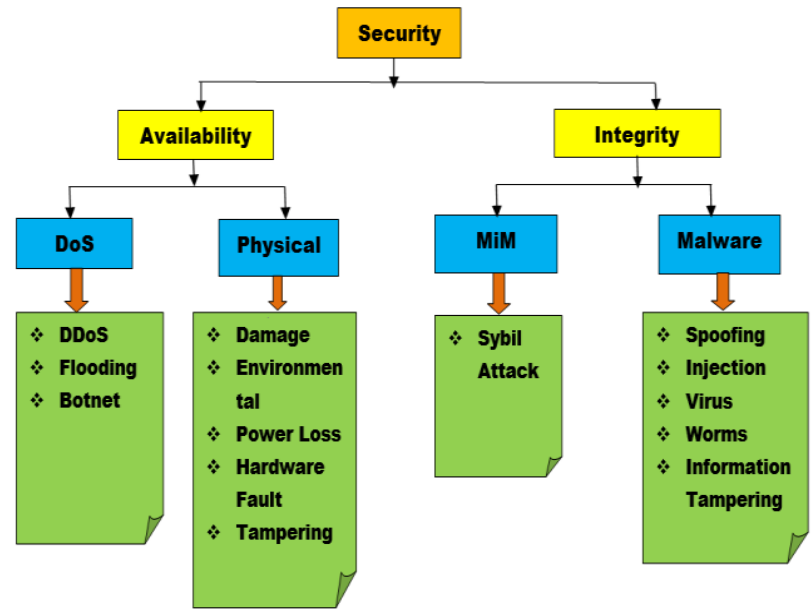

Fig 4: Security threats of IoT/IoMT

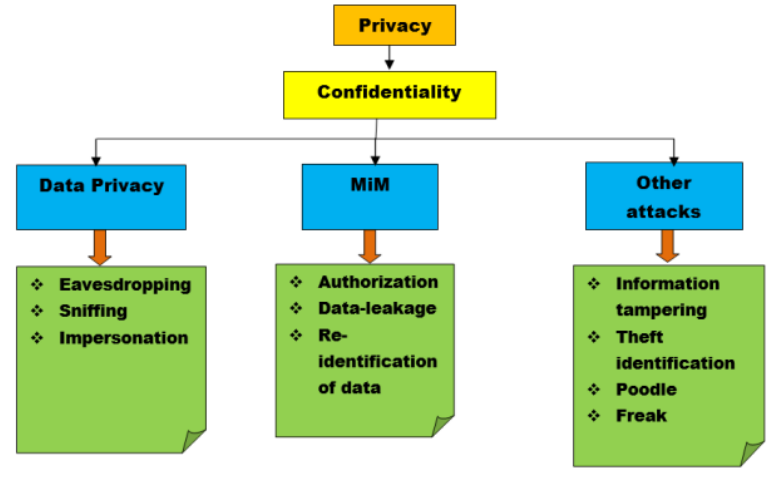

Fig 5: Privacy threats of IoT/IoMT

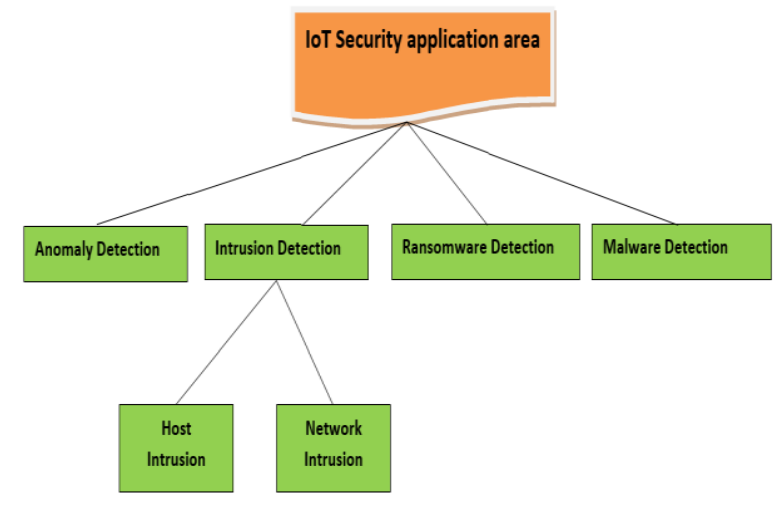

Fig 6: IoT security application area

\section{VARIOUS TYPES OF MALWARE}

Malicious software is shortly known as malware in which is a code or program that is generally offered over a network. It conducts or infects various malicious operations that a hacker or an attacker would like to do. Malware can be separated into various categories according to their functionality features.

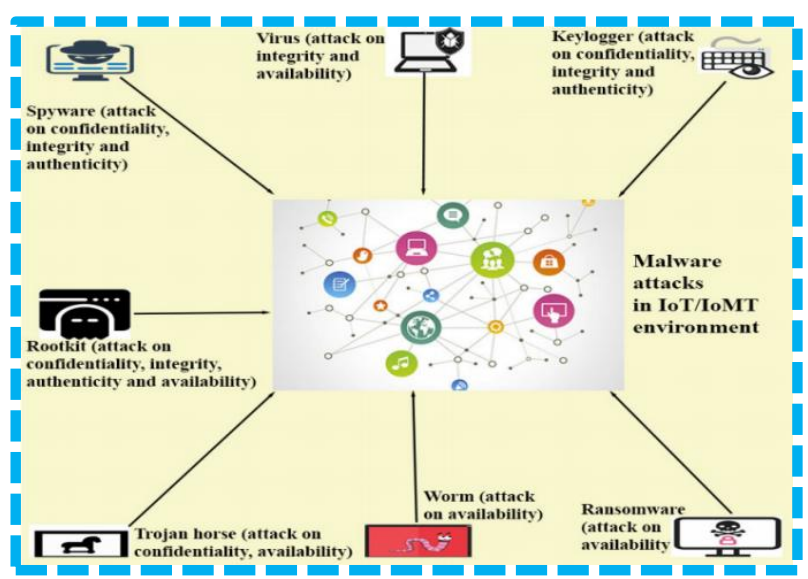

Fig 7: Different kinds of malware

Different kinds of malware (shown in Figure 7) are illustrated below [24-29]:

\subsection{SPYWARE}

Spyware is a kind of malware, which involves by spying the active user without their permission. The malicious types of activities like monitoring, collecting keystrokes, harvesting data such as credit card number, financial data, account credentials, are feasible in the network. It may also infect the software security settings in a device. It can take advantage from the vulnerabilities of the free software and then attach 
itself with several programs.

\subsection{KEYLOGGER}

This type of malicious is a piece of code, which is conducted by a hacker or attacker to track the keystrokes of the operators or users. All information through the keyboard (i.e., their ID, login information, and passwords) have been documented. This malicious attack is stronger than dictionary attack or brute force. The keylogger first attempts to move into a user's Internet based device. It is so terrible that the device cannot be protected with a strong password. Therefore, suggestion for the users is to use multi-factor authentication (i.e., amalgamation of user name, smart card, biometrics data and password).

\subsection{TROJAN HORSE}

This malware pretends itself as a general program to track operators or users into installing after downloading it. In this infected system, it provides the hacker to get opportunity to an authorized remote access. In this system, the hacker can easily steal the data (i.e., credit card information, account number, financial data, password etc.).

\subsection{VIRUS}

This malicious program has a capability of copying itself and deploying to systems. It deploys to each system by including itself to various programs as well as executing the code when a user commences on this infected program. It can steal information, build botnets and damage the host system with the help of this malicious program.

\subsection{WORMS}

It deploys over a network by searching out the weak operating system. It works on the system for damaging their host networks through web servers overload and bandwidth consumption.

\section{RECENT MALWARE ATTACKS}

There are some recent events of malware attacks in IoT/IoMT environment. Some of these are discussed below.

\subsection{MIRAI}

Attacks by Mirai botnet are still going on. Mirai enables monitoring devices running Linux operating systems. These devices can also be conducted as a portion of botnet to carry out different malicious attacks. This malicious program mainly targets smart IoT/IoMT devices, i.e., internet based consumer devices (e.g. home appliances or IP cameras).

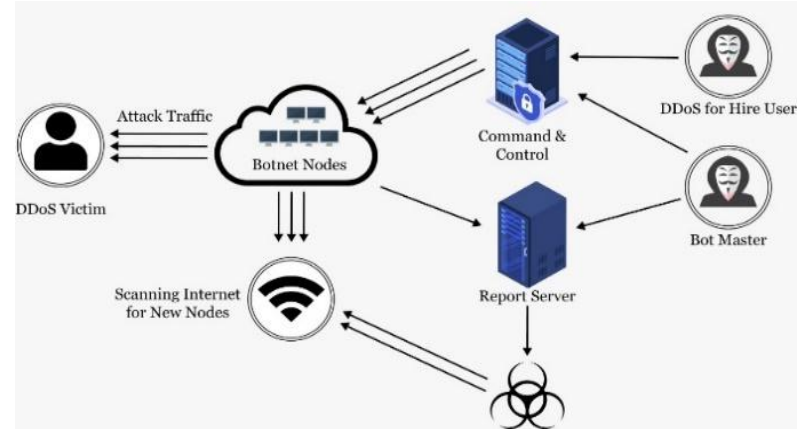

Fig 8: At a glance of MIRAI

Mirai was very active botnet along with the statement of Fortinet in 2018. Moreover, this types of botnets have recently extended some features and these are able to infect IoT/IoMT devices. As of Fortinet, Mirai botnets targeted the IoT or IoMT devices for both unknown and known vulnerabilities. In the botnet, crypto mining exhibits up a crucial change. A hacker can conduct the hardware along with electricity of target's scheme to receive the cryptocurrencies via this types of malware. These typical malicious observances are investigating how to conduct IoMT/IoT botnets to create money [52]-[56].

\subsection{REAPER}

Reaper is a malware that is known as IoTroop. Some researchers of information security created this new botnet with enhanced functionality features in 2017. It can compromise with IoT based smart device rapidly as in contrast to the Mirai botnet. Reaper has various effects as it can overthrow the whole infrastructure rapidly. Mirai corrupts the IoT based smart devices which conduct default passwords and user names. Nevertheless, reaper is more terrible which aims 9 different vulnerabilities in various makers' devices i.e. Linksys, Netgear, and D-Link. Employing this botnet, the hacker can change or vary the malware code to make it more destroying. According to the information served by "Recorded Future". It conducted to attack on several EU banks (i.e., ABN Amro) [56]-[58].

\subsection{ECHOBOT}

Echobot is a kind of malware which is the alteration of Mirai. It was revealed in the year of 2019. This types of malware conducts 26 malicious scripts for expanding its activity. Echobot can put out the advantages of unpatched smart IoT based devices [59]. Ecohobot can create huge number of problems for several applications of the enterprise using these vulnerabilities i.e. weblogic of oracle.

\subsection{EMOTET, GAMUT and NECURS}

Emotet, Gamu and Necurs are used to launch malware attacks in IoT communication environment. At the time of stealing mails from the user's mailbox, Emotet is applied. Emotet is capable to abduct the credentials of SMTP protocol, which is used for mail transfer. Gamut is good at for making spam emails. At preliminary stage, Gamut try to establish a communication with the target device. In order to launch new type of ransomware attack and different digital extortions, Necurs are used.

\section{PERFORMANCE OF DIFFERENT MACHINE LEARNING CLASSIFIERS}

Hackers are becoming very complicated and dangerous with the evolving technology and various new types of malware, making traditional methods of attack-prevention cumbersome. Therefore, protecting an IoT system or Cloud/Fog integrated IoT system becomes more challenging with the limited resources. To help detect these attacks, one of the widely used tools is machine learning (ML) algorithms. Several ML algorithms have proven extremely helpful in mitigating security as well as privacy attacks. The performance of various ML algorithms are summarized in Table 1. Some popular ML algorithms are random forest (RF), decision tree (DT), Naïve Bayes (NB), logistic regression (LR), K nearest neighbor classifier $(\mathrm{kNN})$, support vector machine (SVM), linear discriminant analysis (LDA), etc. In Table 1, TPR and FPR means true positive rate and false positive rate, respectively.

The kNN machine learning classifier achieves better performance and accuracy in the detection of the malware where static features are considered. 
Table 1: Performance comparison of ML algorithms for various malware, intrusion and other types of attack detection

\begin{tabular}{|c|c|c|c|c|}
\hline References & Purpose & Algorithm & Dataset & Results \\
\hline \multirow[t]{5}{*}[30]{} & \multirow[t]{5}{*}{ Intrusion detection } & KNN & \multirow[t]{5}{*}{ Custom } & Accuracy: $99.5 \%$ \\
\hline & & LSVM & & Accuracy: $92.1 \%$ \\
\hline & & DT & & Accuracy: $99.5 \%$ \\
\hline & & $\mathrm{RF}$ & & Accuracy: $99.8 \%$ \\
\hline & & NN & & Accuracy: $98.9 \%$ \\
\hline \multirow[t]{2}{*}[31]{} & \multirow[t]{2}{*}{ Intrusion detection } & RF & \multirow[t]{2}{*}{ NSL-KDD } & Accuracy for DoS attack: $99.67 \%$ \\
\hline & & $\mathrm{J} 48$ & & Accuracy for DoS attack: $99.25 \%$ \\
\hline \multirow[t]{3}{*}[32]{} & \multirow[t]{3}{*}{ Intrusion detection } & DT & \multirow[t]{3}{*}{ Custom } & Accuracy for new attack profile: $64.66 \%$ \\
\hline & & NB & & Accuracy for new attack profile: $57.38 \%$ \\
\hline & & LDA & & Accuracy for new attack profile: $56.00 \%$ \\
\hline \multirow[t]{4}{*}{ [33] } & \multirow{4}{*}{$\begin{array}{l}\text { Malware detection of } \\
\text { Android system }\end{array}$} & DT & - & AUC: $96.4 \%$, Accuracy: $95.4 \%$ \\
\hline & & NB & - & AUC: $91.5 \%$, Accuracy: $86.7 \%$ \\
\hline & & LR & - & AUC: $97.7 \%$, Accuracy: $93.2 \%$ \\
\hline & & PART & - & AUC: $97.0 \%$, Accuracy: $96.3 \%$ \\
\hline$[34]$ & Adeversial attack & $\mathrm{RF}$ & - & Accuracy: $92.79 \%$ (Features: Permission) \\
\hline [35] & $\begin{array}{c}\text { Adeversial attack } \\
\text { detection }\end{array}$ & $\mathrm{RF}$ & - & Accuracy: $94.90 \%$ (Features: Permission) \\
\hline$[36]$ & $\begin{array}{c}\text { Adeversial attack } \\
\text { detection }\end{array}$ & RF & - & $\begin{array}{c}\text { Accuracy: } 92.36 \% \text { (Features: Permission, } \\
\text { API calls) }\end{array}$ \\
\hline [37] & $\begin{array}{c}\text { Adeversial attack } \\
\text { detection }\end{array}$ & KNN & - & $\begin{array}{c}\text { Accuracy: } 97.87 \% \text { (Features: Permission, } \\
\text { API calls) }\end{array}$ \\
\hline$[38]$ & $\begin{array}{l}\text { Impersonate attack } \\
\text { detection }\end{array}$ & KNN & $\begin{array}{c}3987 \text { malware apps } \\
\text { collected from different } \\
\text { sources (McAfee and } \\
\text { Android Malware } \\
\text { Genome Project) }\end{array}$ & $\begin{array}{c}\text { Accuracy: } 99 \%, \text { FPR: } 2.2 \% \text { (Features: API } \\
\text { calls) }\end{array}$ \\
\hline \multirow[t]{4}{*}{ [39] } & \multirow[t]{4}{*}{$\begin{array}{c}\text { Android Malware } \\
\text { detection }\end{array}$} & $\begin{array}{l}\text { Improved } \\
\text { Naïve Bayes }\end{array}$ & \multirow{4}{*}{$\begin{array}{l}\text { Collected from the } \\
\text { Google Play Store and } \\
\text { Chinese App store } \\
(6192 \text { benign, } 5560 \\
\text { malware apps })\end{array}$} & TPR: $98.2 \%$, FPR: $98.2 \%$, Accuracy: $98 \%$ \\
\hline & & NB & & $\begin{array}{c}\text { TPR: } 80.5 \% \text {, FPR: } 80.7 \% \text {, Accuracy: } \\
90.5 \%\end{array}$ \\
\hline & & SVM & & TPR: 95.2\%, FPR: 95.2\%, Accuracy: 95\% \\
\hline & & KNN & & TPR: $75.8 \%$, FPR: $87.5 \%$, Accuracy: $92 \%$ \\
\hline$[40]$ & $\begin{array}{l}\text { Event-aware Android } \\
\text { malware detection }\end{array}$ & $\begin{array}{c}\text { Neural } \\
\text { network }\end{array}$ & $\begin{array}{c}10956 \text { benign samples } \\
\text { in } 2014 \text { from } \\
\text { PlayDrone [53], } 4000 \\
\text { new apps in 2018 from } \\
\text { Play Store [54], and } 28 \\
848 \text { malicious samples } \\
\text { from VirusShare [55]. }\end{array}$ & $\begin{array}{c}\text { For } 2014 \& \text { benign dataset: F1 score- } 99.8 \\
\%, \text { Precision- } 99.1 \% \text {, Recall- } 99.2 \% \\
\text { For } 2018 \& \text { benign dataset: F1 score- } 93.4 \\
\%, \text { Precision- } 92.2 \%, \text { Recall- } 94.7 \%\end{array}$ \\
\hline \multirow[t]{3}{*}[56]{} & \multirow{3}{*}{$\begin{array}{l}\text { Detection of new and } \\
\text { unseen malicious } \\
\text { applications }\end{array}$} & RIPPER & - & Accuracy: $89.36 \%$, FPR: $7.77 \%$ \\
\hline & & NB & - & Accuracy: $97.11 \%$, FPR: $3.80 \%$ \\
\hline & & Multi-NB & - & Accuracy: $96.88 \%$, FPR: $6.01 \%$ \\
\hline
\end{tabular}

\section{RANSOMWARE IN IOT}

Ransomware is a dangerous malware. It hijacks a user's system and steals all of his sensitive data. There are two types of ransomware: (a) Crypto-ransomware and, (b) Lockerransomware. The Crypto-ransomware encrypts user's files and makes them inaccessible to the users [42]. The Lockerransomware locks the user's device interface and demands for ransom to unlock the device. Recent ransomware attacks such as WannaCry and NotPetya have crushed the misconception that a back-up file can protect the digital data of an organization from being hacked [41]. If big institutions and firms were forced to pay money for unlocking file from ransom attack, one can only imagine the situation when a single individual is involved. In other words, when IoT, IoE and ransomware collide and cybercriminals begin to load IoT, 
IoE devices with the dangerous malware, a perfect storm of cyber security arms race will be created. When the malicious code is injected and circulated inside the system, the life cycle of ransomware starts at that time and lasts until the financial claim is shown to the victim. During this lifecycle, a number of activities are conducted in order to hijack the precious files and resources of the IoT user successfully. First, code dropper, mail attachment, or drive by download is utilized for smoothing the path of entering the ransomware's way into the victim's machine $[51,58]$. When appeared, the malicious program in the host machine begins a flow of actions. These actions contain deleting shadow copies, creating a single computer ID, repairing the external IP address, etc., [57]. Ransomware interactions its command and control servers to achieve the encryption key. Then, in the next step, the malicious progress explores for user-related documents or files with particular extensions, i.e., .docx, .pptx, xlsx, .jpg, and .pdf. The encryption step holds place in the next step through passing the position of destined files or documents into a different position and then performing encryption on them. In this step, the encrypted documents or files are renamed. File extension of original files are changed and ransomware extensions are appended to files (e.g. locked, .crypto,_crypt, .crinf, .RDM, .RRK etc.) and the original files are deleted [57]. In the final stage, the financial claim is displayed by the malicious process in which ransom demands to the victim is included in the format of a text file or message of the desktop screen.

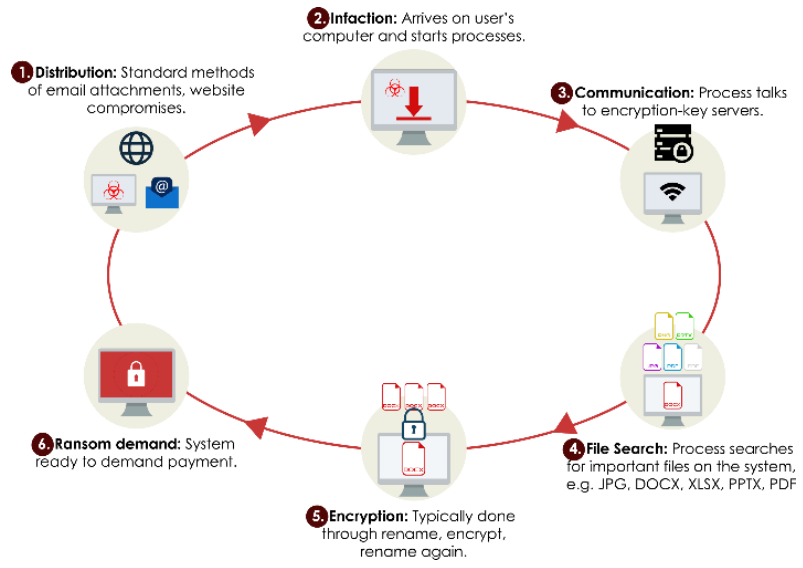

Fig 9: Ransomware follows a number of typical steps to success [57]

Al-rimy conducted a comprehensive survey and assessment of current ransomware related studies in his paper [43]. Even though these researches offered various solutions for ransomware recognition and prevention, there remains various open issues that require advance investigation and research. Here, this paper discusses these research directions and issues which can assist to develop the efficiency and effectiveness of ransomware recognition and prevention solutions [44]. Some existing software tools for detecting, analyzing and predicting ransomware are briefly illustrated in Table 2 .

Table 2: Tools for ransomware detection, classification and analysis

\begin{tabular}{|c|c|c|}
\hline Operation Type & Methods & $\begin{array}{c}\text { Reference } \\
\text { paper }\end{array}$ \\
\hline Static analysis & ApkTool. & {$[45],[46]$} \\
\hline Dynamic & Cuckoo Sandbox. & {$[47-50]$} \\
\hline
\end{tabular}

\begin{tabular}{|c|c|c|}
\hline Operation Type & Methods & $\begin{array}{l}\text { Reference } \\
\text { paper }\end{array}$ \\
\hline analysis & $\begin{array}{l}\text { Microsoft Filesystem } \\
\text { Minifilter Driver. }\end{array}$ & [47] \\
\hline $\begin{array}{c}\text { Features } \\
\text { extraction and } \\
\text { factorization }\end{array}$ & $\begin{array}{c}\text { Term Frequency- } \\
\text { Inverse Document } \\
\text { Frequency (TF-IDF), } \\
\text { Term Frequency } \\
\text { (TF), N-gram, } \\
\text { Frequency-Centric } \\
\text { Model (FCM), } \\
\text { Natural Language } \\
\text { Processing (NLP) }\end{array}$ & $\begin{array}{c}\text { [45], [46], [50], } \\
{[51]}\end{array}$ \\
\hline Classification & $\begin{array}{l}\text { SVM, LR, RF, } \\
\text { Baysian Belief } \\
\text { Network, NB. }\end{array}$ & $\begin{array}{c}\text { [52], [49], [46], } \\
{[50]}\end{array}$ \\
\hline $\begin{array}{c}\text { Similarity } \\
\text { measurement }\end{array}$ & $\begin{array}{l}\text { Structural similarity } \\
\text { (SSIM), Cosine } \\
\text { similarity. }\end{array}$ & {$[45],[47]$} \\
\hline
\end{tabular}

\section{FUTURE SCOPE AND RESEARCH DIRECTION}

In the presence of the recent coronavirus disease 2019 (COVID-19) [62-63], [65-66], the importance of IoMT has greatly increased. Different machine learning [68-69] and deep learning techniques along with sensors [64], image processing [67] and wireless communication techniques [7071] can be used to develop IoMT suitable for detecting and monitoring COVID-19 patients. In this section, several research challenges in hereafter, directions and scopes of malware detection in fog/cloud integrated or IoT related environment.

a) Robust security: Various recent malware detection and prevention methods do not provide full proof security against the new type of malware attacks. Moreover, some of these are attack specific and do not work for other types of attacks at the same time. Therefore, malware detection methods should be robust against multiple malware attacks at the same time.

b) Less computation time and less cost: IoMT/IoT communication environment consists of resourceconstrained devices. This means that smart IoT devices have less storage capacity, short battery life, low computation power. Hence, we cannot use weighty deep learning systems for the malware detection for IoMT/IoT devices. As a result, malware detection methods and prevention mechanisms need to be designed in such a way that the proposed mechanisms must exhibit less computation time and less communication cost without negotiating the security needs.

c) Scalability: IoT is a type of heterogeneous network of different communication standards and applications. These applications have their own requirements and capabilities. In such an environment, we can have the "Electronic Health Records (EHRs)" of certain users that are stored in an IoT-enabled cloud server for further processing. The different devices inside the "Body Area Network (BANs)" produce data and send that to the cloud server. Therefore, it constructs a heterogeneous network of different communicating devices. We need a 
specific type of malware detection mechanism which can protect all types of devices of such kind of communication environment. Hence, more deep research study is needed in this direction.

d) Use of blockchain: The operations of blockchain can be conducted to secure various communication environments. It is because the blockchain operations are decentralized, efficient and transparent. Blockchain operations can also be utilized in efficient detection of the malware in IoT/IoMT environment. In such kind of detection method, we can create a block containing the information about the malicious programs (i.e., malware) to add in the blockchain. Since the blockchain is available to all authorized parties, these parties can have get into the information of the existing malware attacks.

\section{CONCLUSION}

With the advent of modern technologies, it is assumed that the number of smart devices will increase to a great extent. This will lead to the extension of IoT networks. IoT is being used in health care, industry as well as in day to day life. Particularly, IoT can be useful in managing current and future pandemics including COVID-19. With the increase in the number of interconnected devices and the growth of IoT, the number of security vulnerabilities are increasing. Security aspects are in the connected devices, in the data communication process, and in the data storage techniques. Security threats in the form of malware, ransomware, etc., may hinder the progress of IoT. Several security measures are being developed to ensure reliable IoT. This paper advances the current state of the art of security aspects of IoT. This work presents a number of potential security threats in the context of IoT, and discusses about the machine learning algorithms that can be useful in combating these threats. The findings of this paper will help develop more secure IoT networks and provide users secure user experience.

\section{REFERENCES}

[1] Abidi, B.; Jilbab, A.; Haziti, M.E. Wireless sensor networks in biomedical: Wireless body area networks. In Europe and MENA Cooperation Advances in Information and Communication Technologies; Springer: Berlin/Heidelberg, Germany, 2017; pp. 321-329.

[2] Xu, Q.; Ren, P.; Song, H.; Du, Q. Security enhancement for IoT communications exposed to eavesdroppers with uncertain locations. IEEE Access 2016, 4, 2840-2853.

[3] Scuotto, V.; Ferraris, A.; Bresciani, S. Internet of Things: Applications and challenges in smart cities: A case study of IBM smart city projects. Bus. Process Manag. J. 2016, $22,357-367$

[4] Stergiou, C.; Psannis, K.E.; Kim, B.G.; Gupta, B. Secure integration of IoT and cloud computing. Future Generation Computer System. 2018, 78, 964-975.

[5] Truong, H.L.; Dustdar, S. Principles for engineering IoT cloud systems. IEEE Cloud Comput. 2015, 2, 68-76

[6] Dang, L.M.; Piran, M.J.; Han, D.; Min, K.; Moon, H. A Survey on Internet of Things and Cloud Computing for Healthcare. Electronics 2019, 8, 768.

[7] Subrato Bharati, Prajoy Podder, M. R. H. Mondal, Pinto Kumar Paul, "Applications and Challenges of Cloud Integrated IoMT". In "Cognitive Internet of medical things for healthcare: Services and applications", A. E. Hassanien, A. Khamparia, D. Gupta, K. Shankar, A.
Slowik (Eds), to be published by Springer. [In Press].

[8] Shahariar Parvez A.H.M., Robiul Alam Robel M., Rouf M.A., Podder P., Bharati S. (2020) Effect of Fault Tolerance in the Field of Cloud Computing. In: Smys S., Bestak R., Rocha Á. (eds) Inventive Computation Technologies. ICICIT 2019. Lecture Notes in Networks and Systems, vol 98. Springer, Cham.

[9] S. M. Riazul Islam, D. Kwak, M. Humaun Kabir, M. Hossain, and K.-S. Kwak, "The Internet of Things for health care: A comprehensive survey," IEEE Access, vol. 3, pp. 678-708, Jun. 2015.

[10] M. Wazid, A. K. Das, S. Kumari, X. Li, and F. Wu, "Provably secure biometric-based user authentication and key agreement scheme in cloud computing," Secur. Commun. Netw., vol. 9, no. 17, pp. 4103-4119, 2016.

[11] Mondal, M. Rubaiyat Hossain. "Comparison of DCOOFDM, ADO-OFDM, HDC-OFDM and HNC-OFDM for Optical Wireless Communications", Journal of Optical Communications (published online ahead of print), doi: https://doi.org/10.1515/joc-2018-0073.

[12] M. M. H. Mishu and M. R. H. Mondal, "Effectiveness of filter bank multicarrier modulation for $5 \mathrm{G}$ wireless communications," 2017 4th International Conference on Advances in Electrical Engineering (ICAEE), Dhaka, 2017, pp. 319-324, doi: 10.1109/ICAEE.2017.8255374.

[13] M. R. H. Mondal and J. Armstrong, "Analysis of the Effect of Vignetting on MIMO Optical Wireless Systems Using Spatial OFDM," in Journal of Lightwave Technology, vol. 32, no. 5, pp. 922-929, March1, 2014, doi: 10.1109/JLT.2013.2294647.

[14] M. R. H. Mondal and J. Armstrong, "The effect of defocus blur on a spatial OFDM optical wireless communication system," 2012 14th International Conference on Transparent Optical Networks (ICTON), Coventry, 2012, pp. 1-4.

[15] M. I. Khan and M. R. H. Mondal, "Effectiveness of LED index modulation and non-DC biased OFDM for optical wireless communication," 2017 IEEE International Conference on Telecommunications and Photonics (ICTP), Dhaka, 2017, pp. 227-231.

[16] Shahfida Amjad Munni, Rashed Islam, M. Rubaiyat Hossain Mondal, "Performance Evaluation of ASCOOFDM Based LiFi", International Journal of Future Computer and Communication, Vol. 9, Issue 2, pp. 3339, 2020.

[17] Subrato Bharati, Prajoy Podder, "Adaptive PAPR Reduction Scheme for OFDM Using SLM with the Fusion of Proposed Clipping and Filtering Technique in Order to Diminish PAPR and Signal Distortion", Wireless Personal Communication (2020). https://doi.org/10.1007/s11277-020-07323-0.

[18] Subrato Bharati, Prajoy Podder, Niketa Gandhi, Ajith Abraham, "Realization of MIMO Channel Model for Spatial Diversity with Capacity and SNR Multiplexing Gains", International Journal of Computer Information Systems and Industrial Management Applications, Vol. 12, pp. 66-81, 2020.

[19] Rashad J. McFarland, Samuel Bo Olatunbosun, "An Exploratory Study on the use of Internet of Medical Things (IoMT) In the Healthcare Industry and their 
Associated Cybersecurity Risks", Proceedings of the 2019 International Conference on Internet Computing \& Internet of Things, pp. 115-121, 2019.

[20] V. Clincy and H. Shahriar, "IoT malware analysis," in Proc. IEEE $43^{\text {rd }}$ Annu. Comput. Softw. Appl. Conf. (COMPSAC), Milwaukee, WI, USA, vol. 1, Jul. 2019, pp. 920-921.

[21] Mahapatra, S.N., Singh, B.K. \& Kumar, V. A Survey on Secure Transmission in Internet of Things: Taxonomy, Recent Techniques, Research Requirements, and Challenges. Arab J Sci Eng (2020). https://doi.org/10.1007/s13369-020-04461-2

[22] Amaraweera S.P., Halgamuge M.N. (2019) Internet of Things in the Healthcare Sector: Overview of Security and Privacy Issues. In: Mahmood Z. (eds) Security, Privacy and Trust in the IoT Environment. Springer, Cham

[23] E. Shaikh, I. Mohiuddin and A. Manzoor, "Internet of Things (IoT): Security and Privacy Threats," 2019 2nd International Conference on Computer Applications \& Information Security (ICCAIS), Riyadh, Saudi Arabia, 2019, pp. 1-6,

[24] P. Yan and Z. Yan, “A survey on dynamic mobile malware detection," Softw. Qual. J., vol. 26, no. 3, pp. 891-919, 2018.

[25] H. Takase, R. Kobayashi, M. Kato, and R. Ohmura, “A prototype implementation and evaluation of the malware detection mechanism for IoT devices using the processor information," Int. J. Inf. Secur., 2019. doi: 10.1007/s10207-019-00437-y.

[26] A. Azmoodeh, A. Dehghantanha, and K.-K. R. Choo, "Robust malware detection for Internet of (battlefield) things devices using deep Eigenspace learning," IEEE Trans. Sustain. Comput., vol. 4, no. 1, pp. 88-95, Jan./Mar. 2019.

[27] E. M. Rudd, A. Rozsa, M. Günther, and T. E. Boult, “A survey of stealth malware attacks, mitigation measures, and steps toward autonomous open world solutions," IEEE Commun. Surveys Tuts., vol. 19, no. 2, pp. 11451172, 2nd Quart., 2017.

[28] Subrato Bharati, Prajoy Podder, M Rubaiyat Hossain Mondal, Md Robiul Alam Robel, "Threats and Countermeasures of Cyber Security in Direct and Remote Vehicle Communication Systems", Journal of Information Assurance and Security (JIAS) ISSN 15541010, Vol. 15, Issue: 4, pp. 153-164, 2020.

[29] M. Wazid, S. Zeadally, and A. K. Das, 'Mobile banking: Evolution and threats: Malware threats and security solutions," IEEE Consum. Electron. Mag., vol. 8, no. 2, pp. 56-60, Mar. 2019

[30] R. Doshi, N. Apthorpe and N. Feamster, "Machine Learning DDoS Detection for Consumer Internet of Things Devices," 2018 IEEE Security and Privacy Workshops (SPW), San Francisco, CA, 2018, pp. 29-35, doi: 10.1109/SPW.2018.00013.

[31] Nabila Farnaaz, M.A. Jabbar, Random Forest Modeling for Network Intrusion Detection System, Procedia Computer Science, Volume 89, 2016, Pages 213-217,

[32] E. Viegas, A. Santin, L. Oliveira, A. Frana, R. Jasinski, and V. Pedroni, "A reliable and energy-efficient classifier combination scheme for intrusion detection in embedded systems," Computers \& Security, vol. 78, pp. $16-32,2018$.

[33] S. Y. Yerima, S. Sezer, and I. Muttik, "Android Malware Detection Using Parallel Machine Learning Classifiers," 2014 Eighth Int. Conf. Next Gener. Mob. Apps, Serv. Technol., no. Ngmast, pp. 37-42, 2014.

[34] A. Kumar, K. S. Kuppusamy, and G. Aghila, "FAMOUS: Forensic analysis of MObile devices using scoring of application permissions," Future Gener. Comput. Syst., vol. 83, pp. 158-172, Jun. 2018.

[35] U. Pehlivan, N. Baltaci, C. Acartürk and N. Baykal, "The analysis of feature selection methods and classification algorithms in permission based Android malware detection," 2014 IEEE Symposium on Computational Intelligence in Cyber Security (CICS), Orlando, FL, 2014, pp. 1-8.

[36] P. P. K. Chan and Wen-Kai Song, "Static detection of Android malware by using permissions and API calls," 2014 International Conference on Machine Learning and Cybernetics, Lanzhou, 2014, pp. 82-87.

[37] W.-C. Wu and S.-H. Hung, "DroidDolphin: A dynamic android malware detection framework using big data and machine learning," in Proc. Conf. Res. Adapt. Convergent Syst., Oct. 2014, pp. 247-252.

[38] Y. Aafer, W. Du, and H. Yin, DroidAPIMiner: Mining API-Level Features for Robust Malware Detection in Android. New York, NY, USA: Springer, 2014

[39] R. Kumar, X. Zhang, W. Wang, R. U. Khan, J. Kumar, and A. Sharif, "A multimodal malware detection technique for Android IoT devices using various features," IEEE Access, vol. 7, pp. 64411-64430, 2019

[40] T. Lei, Z. Qin, Z. Wang, Q. Li and D. Ye, "EveDroid: Event-Aware Android Malware Detection Against Model Degrading for IoT Devices," in IEEE Internet of Things Journal, vol. 6, no. 4, pp. 6668-6680, Aug. 2019,

[41] S. R. Zahra and M. Ahsan Chishti, "RansomWare and Internet of Things: A New Security Nightmare," 2019 9th International Conference on Cloud Computing, Data Science \& Engineering (Confluence), Noida, India, 2019 , pp. 551-555.

[42] Ayesha Naseer, Riffat Mir, Muhammad Aleem, Windows-based Ransomware: A Survey, Journal of Information Assurance and Security. ISSN 1554-1010 Volume 15 (2020) pp. 107-125

[43] Bander Ali Saleh Al-rimy, Mohd Aizaini Maarof, Syed Zainuddin Mohd Shaid, Ransomware threat success factors, taxonomy, and countermeasures: a survey and research directions, Computers \& Security (2018), https://doi.org/10.1016/j.cose.2018.01.001

[44] K. P. Subedi, D. R. Budhathoki and D. Dasgupta, "Forensic Analysis of Ransomware Families Using Static and Dynamic Analysis," 2018 IEEE Security and Privacy Workshops (SPW), San Francisco, CA, 2018, pp. 180185, doi: 10.1109/SPW.2018.00033.

[45] Andronio, N., Zanero, S., and Maggi, F. (2015). HELDROID: Dissecting and detecting mobile ransomware. In H. Bos, G. Blanc and F. Monrose (Eds.), 
18th International Symposium on Research in Attacks, Intrusions, and Defenses, RAID 2015 (Vol. 9404, pp. 382-404): Springer Verlag.

[46] Maiorca, D., Mercaldo, F., Giacinto, G., Visaggio, C. A., and Martinelli, F. (2017). R-PackDroid: API packagebased characterization and detection of mobile ransomware. Paper presented at the Proceedings of the Symposium on Applied Computing.

[47] Kharraz, A., Robertson, W., Balzarotti, D., Bilge, L., and Kirda, E. (2015). Cutting the gordian knot: A look under the hood of ransomware attacks. In F. Maggi, M. Almgren and V. Gulisano (Eds.), 12th International Conference on Detection of Intrusions and Malware, and Vulnerability Assessment, DIMVA 2015 (Vol. 9148, pp. 3-24): Springer Verlag.

[48] Scaife, N., Carter, H., Traynor, P., and Butler, K. R. (2016). CryptoLock (and Drop It): Stopping Ransomware Attacks on User Data.

[49] Sgandurra, D., Muñoz-González, L., Mohsen, R., and Lupu, E. C. (2016). Automated Dynamic Analysis of Ransomware: Benefits, Limitations and use for Detection. arXiv preprint arXiv:1609.03020.

[50] Al-rimy, B. A. S., Maarof, M. A., and Shaid, S. Z. M. (2017). A 0-Day Aware Crypto- Ransomware Early Behavioral Detection Framework. Paper presented at the International Conference of Reliable Information and Communication Technology, 758- 766.

[51] Mbol, F., Robert, J.-M., and Sadighian, A. (2016). An Efficient Approach to Detect TorrentLocker Ransomware in Computer Systems. In S. Foresti and G. Persiano (Eds.), Cryptology and Network Security: 15th International Conference, CANS 2016, Milan, Italy, November 14-16, 2016, Proceedings (pp. 532-541). Cham: Springer International Publishing.

[52] Ahmadian, M. M., and Shahriari, H. R. (7-8 Sept. 2016). 2entFOX: A framework for high survivable ransomwares detection. Paper presented at the 2016 13th International Iranian Society of Cryptology Conference on Information Security and Cryptology (ISCISC), 79-84.

[53] N. Viennot, E. Garcia and J. Nieh, "A measurement study of Google play", Proc. ACM SIGMETRICS, vol. 42, no. 1, pp. 221-233, 2014.

[54] Google Play Store, Mar. 2019, [online] Available: https://play.google.com/store.

[55] VirusShare, Mar. 2019, [online] Available: https://virusshare.com/.

[56] M. G. Schultz, E. Eskin, F. Zadok and S. J. Stolfo, "Data mining methods for detection of new malicious executables," Proceedings 2001 IEEE Symposium on Security and Privacy. S\&P 2001, Oakland, CA, USA, 2001, pp. 38-49.

[57] McAfee, L. (2016). Understanding Ransomware and Strategies to Defeat It. In I. Security (Ed.)

[58] Kim, D., Soh, W., and Kim, S. (2015). Design of Quantification Model for Prevent of Cryptolocker. Indian Journal of Science and Technology, 8(19).

[59] M. Korolov. What is a Botnet? When Armies of Infected
IoT Devices Attack. Accessed: June, 2020. [Online]. Available: https://www.csoonline.com/ article/3240364/ what-is-a-botnet.html

[60] https://www.quora.com/What-advancements-can-we-doin-a-healthcare-monitoring-system-using-IoT (last accessed July 21, 2020)

[61] https://www.iiot-center.org/ (last accessed July 21, 2020)

[62] Khanam F., Nowrin I., and Mondal M. R. H., "Data Visualization and Analyzation of COVID-19", Journal of Scientific Research and Reports, vol. 26, no. 3, pp. 4252, Apr. 2020.

[63] Mondal M. R. H., Bharati S., Podder P., Podder P., "Data analytics for novel coronavirus disease", Informatics in Medicine Unlocked, Volume 20, 2020, 100374, ISSN 2352-9148, https://doi.org/10.1016/j.imu.2020.100374.

[64] Bharati S., Podder P., Mondal M.R.H., "Hybrid deep learning for detecting lung diseases from X-ray images", Informatics in Medicine Unlocked, Volume 20, 2020, 100391, ISSN 2352-9148, https://doi.org/10.1016/j.imu.2020.100391.

[65] Subrato Bharati, Prajoy Podder, M. R. H. Mondal, Priya Podder, Utku Kose, "A Review on Epidemiology, Genomic Characteristics, Spread and Treatments of COVID-19". In "Data Science for COVID-19", Utku Kose, Deepak Gupta, Victor H.C. de Albuquerque, Ashish Khanna (Eds), Elsevier. [In Press].

[66] Prajoy Podder, Subrato Bharati, M. R. H. Mondal, Utku Kose, "Application of Machine Learning for the Diagnosis of COVID-19". In "Data Science for COVID19", Utku Kose, Deepak Gupta, Victor H.C. de Albuquerque, Ashish Khanna (Eds), Elsevier. [In press].

[67] M. A. Kabir and M. R. H. Mondal, "Edge-Based and Prediction-Based Transformations for Lossless Image Compression", Journal of Imaging, vol. 4, no. 5, DOI: 10.3390/jimaging4050064, May 2018.

[68] Bharati S., Podder P., Mondal M.R.H., "Visualization and prediction of energy consumption in smart homes", International Journal of Hybrid Intelligent Systems, IOS Press, 2020. DOI: 10.3233/HIS-200283.

[69] S. Bharati, P. Podder, and M. R. H. Mondal, Diagnosis of Polycystic Ovary Syndrome Using Machine Learning Algorithms. Presented at 2020 IEEE Region 10 Symposium (TENSYMP), 5-7 June 2020, Bangladesh.

[70] Kumar V., Mishra B.K., Mazzara M., Thanh D.N.H., Verma A. (2020) Prediction of Malignant and Benign Breast Cancer: A Data Mining Approach in Healthcare Applications. In: Borah S., Emilia Balas V., Polkowski Z. (eds) Advances in Data Science and Management. Lecture Notes on Data Engineering and Communications Technologies, vol 37. Springer, Singapore.

[71] S. Bharati, P. Podder, M. R. H. Mondal, "Artificial Neural Network Based Breast Cancer Screening: A Comprehensive Review", International Journal of Computer Information Systems and Industrial Management Applications, MIR Labs, USA, vol. 12 (2020), pp. 125-137, May 2020 